\title{
Research on the compression method for domestic air conditioner with water vapor as the refrigerant
}

\author{
Xue Xing ${ }^{1, a}$, Li Sheng ${ }^{2, b}$, Wang $\mathrm{An}^{3, \mathrm{c}}$ \\ ${ }^{1,2,3}$ Guilin university of electronic technology, Guangxi province, China \\ a email: 290684211@qq.com, bemail: 913820020@qq.com, cemail:1030913258@qq.com
}

Keywords: air conditioner, water vapor, negative pressure, compression method, vacuum pump

\begin{abstract}
Domestic air conditioners generally use Freon as the refrigerant, which results in serious damages to ozone layer and exacerbates greenhouse effect badly, using water vapor as the refrigerant can avoid the problems above, but there exist series of technical problems in domestic air conditioners using water vapor as the refrigerant such as high compression ratio, large volume flow, working under the negative pressure condition. Via the comparative analysis of the volume compressor, the speed compressor and the volume vacuum pump, the compression method for domestic air conditioners using water vapor as the refrigerant is put forward.
\end{abstract}

\section{Introduction}

Air conditioners have become imperative in people's daily lives, the demand of domestic air conditioner market of China has exceeded 100 million units in 2013 and is still increasing. Air conditioning systems widely adopt Freon refrigerants, its extensive use has brought a series of environmental problems, some Freon refrigerants damage the ozone layer seriously, the ozone layer plays the role of filtering on ultraviolet, excessive ultraviolet will increase the chance of skin cancer, some Freon refrigerants exacerbate the greenhouse effect badly, leading to the deterioration of the ecological environment eventually, thus looking for an alternative refrigerant becomes the common problem facing the world ${ }^{[1]}$. In the process of finding alternative refrigerants, water has attracted people's attention, although water has long been used in the field of absorption and jet refrigeration, the study in vapor compression refrigeration just starts recently due to the pressure of environmental protection $^{[9]}$.

\section{Principle of vapor compression refrigeration}

Domestic air conditioners generally adopt vapor compression refrigeration, mainly composed by the compressor, the condenser, the throttle and the evaporator.

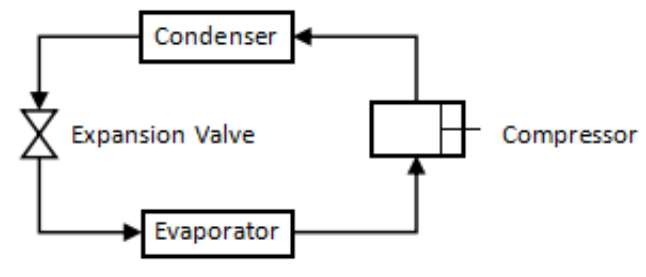

Figure.1 Schematic diagram of vapor compression refrigeration

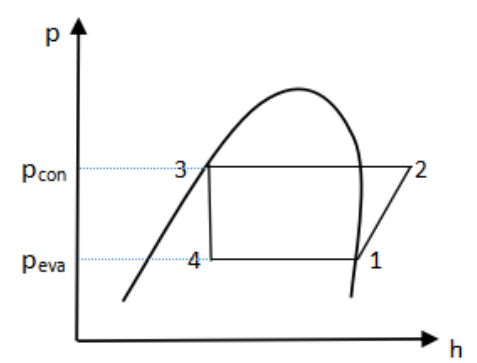

Figure.2 Pressure enthalpy diagram of vapor compression refrigeration

The theoretical cycle consists of an isobaric endothermic process (1-2), an adiabatic 
compression process (2-3), an isobaric exothermic process (3-4), and an adiabatic throttle process (4-1). The low-pressure gaseous refrigerant from the evaporator is adiabatically compressed into high-pressure gaseous state in the compressor (1-2), and cooled into liquid by outdoor air in the condenser (2-3), then getting into the evaporator through an adiabatic throttle process (3-4), absorbing the external air heat thus achieving refrigeration in the evaporator (4-1), finally reaching the compressor to start the next cycle.

\section{Comparative analysis of water vapor and other refrigerants}

In this research, the evaporation temperature is $10^{\circ} \mathrm{C}$, the compressor suction temperature is $12^{\circ} \mathrm{C}$, the difference of condensation temperature and air inlet temperature of the air-cooled condenser can be $10^{\circ} \mathrm{C}^{[2]}$, the condensation temperature is thus $45^{\circ} \mathrm{C}$. Water latent heat is $2500 \mathrm{KJ} / \mathrm{Kg}$, due to the large volume flow of water vapor, to reduce the evaporator volume and to maintain the refrigerating requirement, this paper takes the domestic air conditioner with a cooling capacity of $2500 \mathrm{~W}$ as the research object, which can meet the refrigerating requirement of a $20 \mathrm{~m}^{2}$ room. Via a theoretical cycle without considering the subcooling and overheating degree, parameters of water and other refrigerants under the selected working condition are listed in the table below:

Table.1 Comparison of water with other refrigerants

\begin{tabular}{|c|c|c|c|c|c|c|c|c|c|c|}
\hline refrigerant & ODP & GWP & $\begin{array}{l}\text { Safety } \\
\text { group }\end{array}$ & $\begin{array}{c}\text { compress } \\
\text { ratio }\end{array}$ & $\begin{array}{l}\text { refrigeration } \\
\text { n capacity } \\
(\mathrm{kJ} / \mathrm{kg})\end{array}$ & $\begin{array}{c}\text { outlet } \\
\text { temperature } \\
{ }^{\circ} \mathrm{C}\end{array}$ & $\begin{array}{c}\text { specific } \\
\text { volume } \\
\mathrm{v}_{1}\left(\mathrm{~m}^{3} / \mathrm{kg}\right)\end{array}$ & $\begin{array}{l}\text { volume } \\
\text { flow } \\
\mathrm{v}_{\mathrm{r}}(\mathrm{L} / \mathrm{s})\end{array}$ & $\begin{array}{l}\text { evaporation } \\
\text { pressure } \\
\mathrm{p}_{1}(\mathrm{kPa})\end{array}$ & $\begin{array}{c}\text { condensation } \\
\text { pressure } \\
\mathrm{p}_{2}(\mathrm{kPa})\end{array}$ \\
\hline R22 & 0.03 & 1900 & A1 & 2.6 & 150 & 60 & 0.03 & 0.5 & 670 & 1730 \\
\hline R123 & 0.02 & 93 & B1 & 3.6 & 140 & 45 & 0.3 & 5.4 & 50 & 180 \\
\hline R134a & 0 & 1300 & A1 & 2.8 & 135 & 56 & 0.05 & 0.9 & 410 & 1150 \\
\hline R407c & 0 & 0.05 & B1 & 2.8 & 145 & 53 & 0.04 & 0.7 & 615 & 1725 \\
\hline R717 & 0 & 0 & B2 & 2.9 & 1050 & 85 & 0.2 & 0.5 & 610 & 1780 \\
\hline R718 & 0 & 0 & A1 & 7.8 & 2500 & 215 & 106 & 106 & 1.2 & 9.6 \\
\hline
\end{tabular}

Safety group: A low toxicity, B middle toxicity, C high toxicity; 1 not flammable, 2 flammable, 3 explosive ${ }^{[11]}$.

compression ratio: $\xi=\frac{\mathrm{p}_{2}}{\mathrm{p}_{1}}$, unit refrigeration capacity: $\mathrm{q}_{0}=\mathrm{h}_{1}-\mathrm{h}_{4}$, volume flow: $V_{\mathrm{r}}=\frac{\phi_{0}}{\mathrm{q}_{0}} \mathrm{v}_{1}$

Taking the loss of water vapor into account, the volume we choose is $120 \mathrm{~L} / \mathrm{s}$, from Table 1 we can see the main features of water vapor compression refrigeration: high compression ratio, but the difference between condensation pressure and evaporation pressure is not high; big volume flow, although the latent heat of water is high, the density of water vapor is low, the volume flow of water vapor is thus relatively big; this air conditioning system is working under negative pressure, while other refrigerants are all working under high pressure.

\section{Comparative analysis of compressors}

There are two types of compressors for air conditioners, the volume compressor and the speed compressor, typical compression methods are selected from the two types, the volume compression method we choose are reciprocating and rolling rotor compressor, the speed compression method we choose are radial and axial compressor.

Reciprocating compressor. The working process of reciprocating compressor contains three stages, the suction, the compression and the exhaust. During the suction stage, the piston moves from side a to the right, the pressure in the cylinder decreases, when reaching the suction valve pressure, the suction valve opens and the low-pressure gas was sucked, the suction stage ends when the piston moves to side b; during the compression stage, the piston moves from side $b$ to the left, the cylinder 
pressure increases; when the cylinder pressure is higher than the exhaust valve pressure, the exhaust valve opens and the high temperature and high pressure gas discharges, the discharge stage ends until the piston moved back to side a.

Formula of the volume of Inhale gas: $V_{\mathrm{g}}=\frac{\pi}{240} \cdot D^{2} \cdot \mathrm{L} \cdot \mathrm{z} \cdot \mathrm{n}$

For piston compressors, setting the ratio of piston stroke $\mathrm{L}$ and cylinder diameter D to 0.4-0.6 can reduce the inertia force and frictional resistance, when increasing the number of cylinders $\mathrm{z}$, the rotating speed $n$ will be correspondingly reduced, so the number of cylinders $\mathrm{z}$ we choose is 1 , the volume flow of water vapor we need is $120 \mathrm{~L} / \mathrm{s}$, after calculation we find that even if the rotating speed reaches $3000 \mathrm{r} / \mathrm{min}$, the highest rotating speed commonly used, the piston diameter is still more than $100 \mathrm{~cm}$, when taking the volumetric efficiency into account, the piston diameter will be larger, therefore the piston compressor can not meet the compression requirements of domestic air conditioner with water vapor as refrigerant.

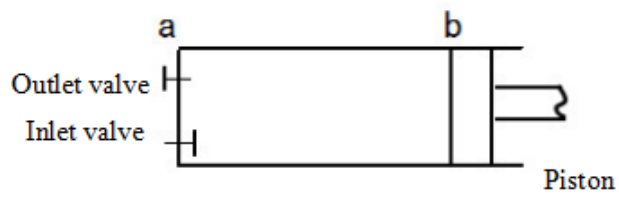

Figure.3 Schematic diagram of reciprocating compressor

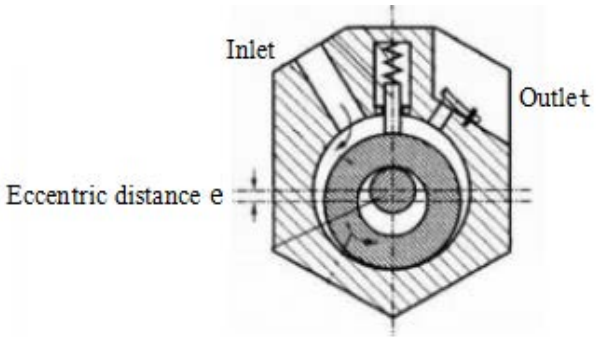

Figure.4 Schematic diagram of rolling rotor compressor

Rolling rotor compressor. A suction hole and an exhaust hole are in the upper part of the cylinder, the exhaust valve can prevent gas backflow, the principal spindle with an eccentric wheel is at the center of the cylinder, the eccentric distance is e, the rotor moves along the surface of the cylinder as the eccentric wheel rotates, the slide contacts with the inner surface of cylinder under centrifugal force, crescent working cavity formed by the rotor and the cylinder is divided into two parts by the slide, the volume of the two parts changes with the variation of the rotor position, the compression process of the gas is thus completed.

Formula of the volume of Inhale gas: $V_{\mathrm{g}}=\frac{\pi}{4}\left(D^{2}-\mathrm{d}^{2}\right) \cdot \mathrm{H} \cdot \mathrm{n}$

For rolling rotor compressor, the cylinder diameter is $\mathrm{D}$, the rotor diameter $\mathrm{d}$ we choose is $0.5 \mathrm{D}$, the axial length $\mathrm{H}$ is $1.2 \mathrm{D}$, substituting the steam volume flow $120 \mathrm{~L} / \mathrm{s}$ into the formula, results show that the diameter of the cylinder is also large, therefore the rolling rotor compressor can not meet the requirements of water vapor compression for domestic air conditioner use.

Centrifugal compressor. After entering the centrifugal impeller, the refrigerant obtain kinetic energy and pressure energy via centrifugal force generated by the high-speed rotation of the impeller, outside the impeller is the diffuser, which converts the kinetic energy into pressure energy of the air flow, and high pressure gas is thus obtained.

The passages of the compressor is chosen via the compression ration, the compression ratio of water vapor under the chosen working condition is 7.8, to reduce the diameter of the impeller, we choose 3 passages compression with 2 intercoolings. In order to guarantee a certain compression margin and ensure the compression efficiency of the third impeller, the compression ration we choose of each passage respectively 2.1, 2 and 1.9. The compression work is determined via the compression ration firstly, the exit circumferential velocity of the gas flow is determined via the 
tangential velocity coefficient of the gas flow and the number of impellers then, the rotation speed of the impeller is determined via the exit circumferential velocity of the gas flow and volume of the inlet gas flow finally, related formulas and parameters are shown in the following table:

Table 2 Related formulas and parameters of radial compressor

\begin{tabular}{|c|c|}
\hline Related fo & \\
\hline $\begin{array}{l}\text { Polytropic compression work: } \\
\qquad \mathrm{h}_{\mathrm{pol}}=\frac{\mathrm{k}}{\mathrm{k}-1} \eta_{\mathrm{pol}} R T_{\mathrm{in}_{\mathrm{n}}}\left(\xi^{\frac{\mathrm{k}-1}{\mathrm{k}}}-1\right) \\
\text { Euler formula: } \mathrm{h}_{\mathrm{pol}}=\mathrm{i} \psi_{\mathrm{pol}} \mathrm{u}_{2}{ }^{2} \\
\text { The Mach number: } M_{\mathrm{u} 2}=\frac{\mathrm{u}_{2}}{\sqrt{\mathrm{k} R T_{i n}}} \\
\text { Impeller rotation speed: }\end{array}$ & $\begin{array}{l}\mathrm{K}-\text {-Isentropic index, } 1.3 ; \\
\eta_{\text {pol }} \text { - Polytropic efficiency, } 0.84 ; \\
\mathrm{R}-\text { Gas constant of water vapor, } 462 \mathrm{~J} /(\mathrm{Kg} \cdot \mathrm{K}) ; \\
\mathrm{T}_{\text {in }}-\text { - Inlet temperature of gas flow; } \\
\mathrm{i}-\text {-The number of impellers; } \\
\psi_{\text {pol }} \text { - Tangential velocity coefficient of gas flow, } 0.45- \\
0.55 ; \\
\mathrm{k}_{\mathrm{v} 2}-\text { Ratio of inlet and outlet gas specific volume; } \\
\tau_{2}-\text { - Impeller outlet section coefficient; } \\
\frac{\mathrm{b}_{2}}{D_{2}}-\text { Relative width of impeller outlet; } \\
\Phi_{2 \mathrm{r}}-\text { Radial velocity coefficient of gas flow; }\end{array}$ \\
\hline
\end{tabular}

From the formulas we can see that the larger the circumferential velocity of the impeller outlet $\mathrm{u}_{2}$ is, the more energy the gas flow can obtain, but on one hand $\mathrm{u}_{2}$ is limited by the strength of the impeller material, it can't exceed $275 \mathrm{~m} / \mathrm{s}^{[2]}$, on the other hand, $\mathrm{u}_{2}$ is affected by the Mach number $M_{u 2}$, the range of $M_{u 2}$ is 1.3-1.5, when the local sound speed is high, the restriction is the strength of the impeller material; when the local sound speed is low, the restriction is the Mach number ${ }^{[3]}$.

The inlet temperature of the compressor is $12^{\circ} \mathrm{C}$, the corresponding sound speed of water vapor is $414 \mathrm{~m} / \mathrm{s}$, therefore the restriction is the strength of the impeller material, one way to reduce the rotation speed is to reduce $u_{2}$, to reduce $u_{2}$ we must improve the number of the impellers, the other way is to improve the volume of water vapor, calculation results show that even if we increase the number of impellers to 4 , the quality of water vapor to $3 \mathrm{~g} / \mathrm{s}$, the rotation speed of the impeller still exceeds $400 \mathrm{r} / \mathrm{s}$, which is far beyond the rotation speed of the compressor commonly used.

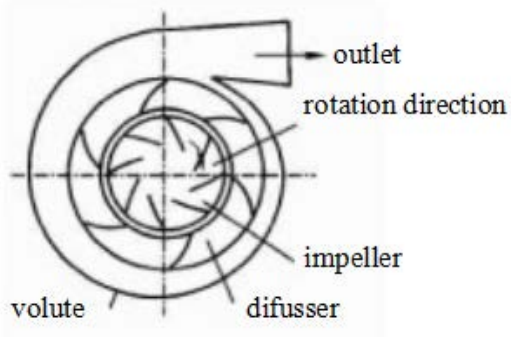

Figure.5 Schematic diagram of radial compressor

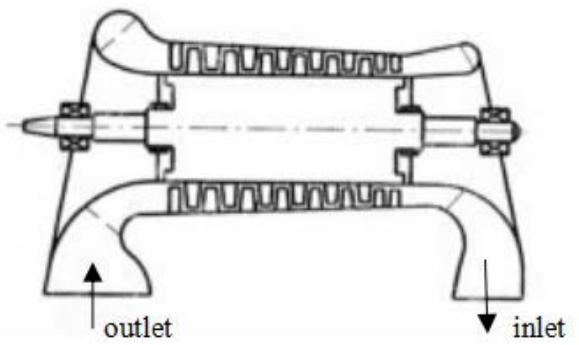

Figure.6 Schematic diagram of axial compressor

Axial compressor. In axial compressors, the gas moves along the axis of rotation, the kinetic energy of the gas flow is increased via the rotation of the impeller, and converts into pressure energy via the diffuser, the pressure raise of the axial is higher than that of the radial, the efficiency is also higher.

The rotation speed of the impeller can be determined after selecting the inlet axial speed of the gas flow and the tip circumferential velocity of the impeller, the number of the impellers can be determined after calculating the ratio of the isentropic energy head of the compressor and the first 
impeller, related formulas and parameters are shown in the table below:

Table 3 Related formulas and parameters of axial compressor

\begin{tabular}{|c|c|}
\hline Related $\mathrm{f}$ & Related pa \\
\hline 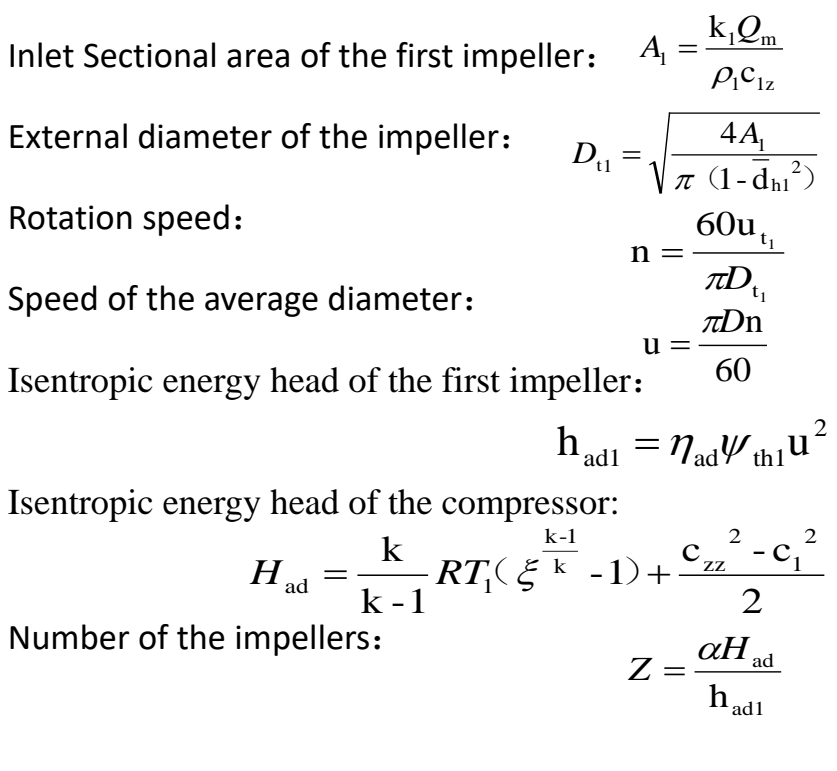 & 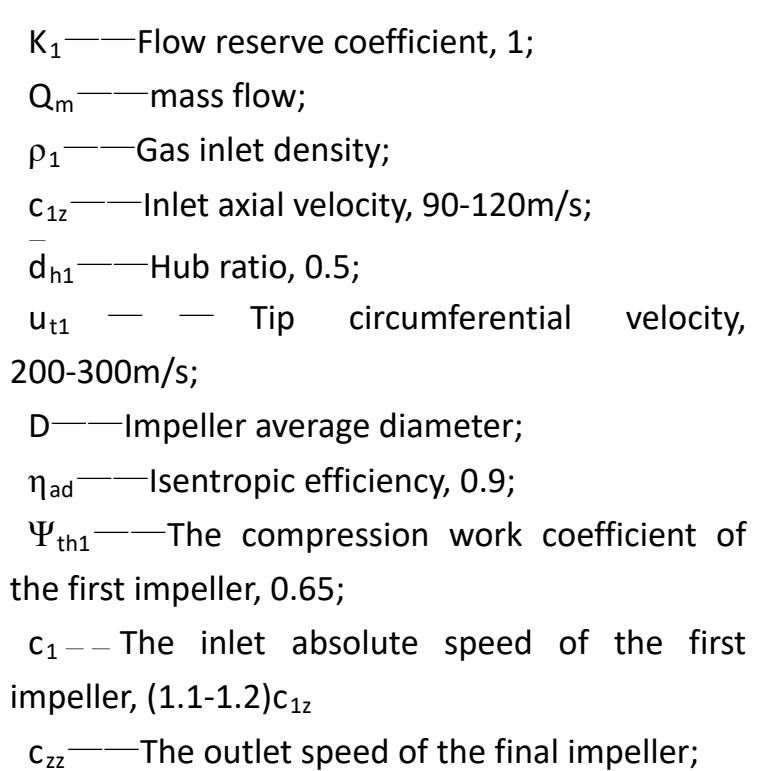 \\
\hline
\end{tabular}

From the formulas above we can see that the impeller rotation speed is proportional to the tip circumferential velocity, in order to reduce the impeller rotation speed, the tip circumferential velocity we choose is $200 \mathrm{~m} / \mathrm{s}$, substituting the related parameters of water vapor into the formulas above, the impeller external diameter we obtain is $47 \mathrm{~cm}$ and the impeller average diameter is $35 \mathrm{~cm}$, the rotation speed is $135 \mathrm{r} / \mathrm{s}$, although compared with the centrifugal impeller it has greatly reduced, however, the number of the impellers has also reached 20.

In the study of water vapor compression refrigeration, the double stage centrifugal and four stage axial compressor have already appeared overseas, although the difference between the working condition they choose and that in this paper is not big, the refrigerating capacity they choose is about $200 \mathrm{KW}$, the volume flow of water vapor is thus relatively big, it's better for them to choose the speed compressor, while the volume flow of water vapor in this research is still relatively small, so there is no need to choose the speed compressor.

\section{Comparative analysis of volumetric vacuum pump}

Rotary vane vacuum pump. An eccentric rotor exists in the pump body, the rotor is tangent (a small gap still exists) to the upper surface of the pump chamber, there are 2 rotary vanes equipped with spring in the rotor groove, the rotor drives the rotary vanes, the top of the rotary vane keeps in touch with the pump internal surface under the centrifugal force, the 2 rotary vanes divide the pump chamber into 3 parts. The compression process is 1-2, the space between the 2 vanes gradually decreases and the gas is thus compressed; the exhaust process is 2-3, when the gas pressure between the vane and the exhaust hole reaches the exhaust pressure, the exhaust valve open and the gas of high pressure is exhausted; the suction process is $3-4$, the space between the vane and the suction hole gradually increases and the gas of low pressure is sucked, and the cycle is completed.

Rotary vane vacuum pump can work in the range of $100-1.33 \times 10^{-2} \mathrm{~Pa}$, the pumping speed of $\mathrm{X}$-150 rotary vane vacuum pump reaches $150 \mathrm{~L} / \mathrm{s}$, which can meet the compression requirement of the volume of water vapor, the rotation speed is only $360 \mathrm{r} / \mathrm{min}$, however, the pump internal surface 
and the rotary vane is lubricated by oil, when meeting water, the emulsification phenomenon happens, therefore this kind of vacuum pump can’t complete the compression of water vapor.

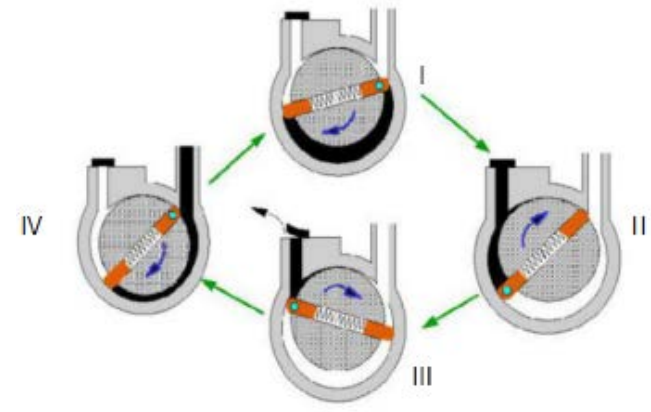

Figure.7 Principle of rotary vane vacuum pump



Figure.8 Principle of water ring vacuum pump

Water ring vacuum pump. A moderate amount of water is in the pump body as the working fluid, when the impeller rotates clockwise, due to the centrifugal force, the water formed a closed ring with similar thickness,the upper part of the water ring is tangent to the impeller hub,the lower part of the water ring touches the top of the impeller,a crescent space between the impeller wheel and the water ring is thus formed,and the space is divided into several small parts,the volume of the right parts increases as the impeller rotates, the volume of the left parts decreases as the impeller rotates.

Water ring vacuum pump adopts water as working fluid, so the gas is not polluted by oil, although the pumping speed of some models of water ring pump can meet the requirements of the volume flow of water vapor, however, the ultimate vacuum of a single stage water ring vacuum pump only reaches 30torr, the ultimate vacuum of a 2 stage water ring vacuum pump only reaches 15 torr $^{[5]}$,in addition, water ring pump generates noise, therefore this kind of vacuum pump also can't complete the compression of water vapor.

Roots vacuum pump. The compression work of Roots vacuum pump is relied on a couple "8" type rotors, the suction process is fromIto II, the gas gets in the pump chamber from the suction port, the suction process ends at III, the volume of the inhaled gas is $\mathrm{V}_{0}$ and formed by the rotor and the pump body, the inhaled gas communicates with the exhaust port, due to the higher pressure of the gas at the exhaust port, part of gas at the exhaust port backflow, the rotor continuous rotation enforce the gas exhausted from the exhaust port, the compression process is thus completed, at this time the other side of the rotor communicates with the suction port, the suction process starts again, one rotor can inhale a $2 \mathrm{~V}_{0} / \mathrm{r}$ volume of gas.

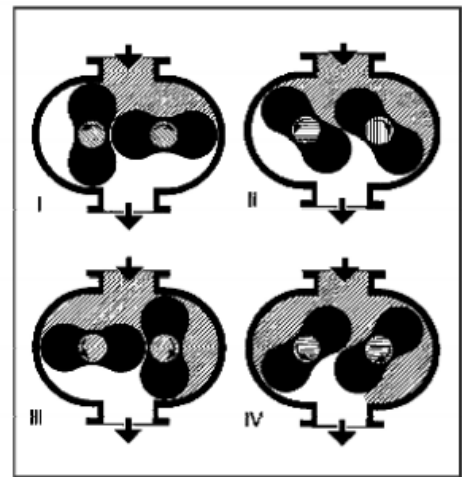

Figure.9 Principle of Roots vacuum pump

The pump cavity and the rotor needs no lubrication, so the gas is not polluted by oil, Roots pump has a large pumping speed in the range of $1-10^{4} \mathrm{~Pa}^{[5]}$, which can meet the pressure demand of water vapor compression. The pumping speed of ZJ-150 Roots pump reaches 150L/s, which can meet the 
volume demand of water vapor compression; the rotation speed is less than $3000 \mathrm{r} / \mathrm{min}$, which can be achieved by commonly used motor; the highest pressure difference of inlet and outlet is 6000Pa, lower than that of water vapor, therefore a double stage Roots pump is imperative to complete the compression of water vapor, or else, reducing the refrigerating capacity to $1750 \mathrm{KW}$, the volume flow of water vapor is 70L/s at this time, ZJ-70 Roots pump can complete its compression work.

\section{Summary}

The key technology of domestic air conditioner with water vapor as the refrigerant is the compression, there are two main problems to be solved, one is the compression of large volume, the other is the compression under under vacuum condition, the volumetric and speed compressor can't solve the two problems above, rotary vane pump results in oil pollution, water ring pump can't reach the pressure needed, therefore Roots pump is the ideal choice for domestic air conditioner with water vapor as the refrigerant.

\section{References}

[1] Lian Leming,Tan Yufei,Wu Jiazheng.Engineering Thermodynamics[M].China Building Industry Press,2007.

[2] Yan Qisen,Shi Wenxing,Tian Changqing.Refrigeration technology for air conditioning[M].China Building Industry Press,2010.

[3] Huang Zhongyue,Wang Xiaofang.Turbo compressor[M].Chemical Industry Press,2004.

[4] Cheng Xinde.Vane pumps·fans·compressors[M].Machinery Industry Press,2011.

[5] Yang Naiheng. Vacuum acquisition equipment[M].Metallurgical Industry Press,1987.

[6] Ren Jinlu.Chiller for water vapor compression[J].Refrigeration and air conditioner, 2008.8:53-59.

[7] Yuan Xiugan, Yuan Weixing,Yu Zhiqiang. Performance research of water vapor compression refrigerant[J].Refrigeration Journal,2003,3:16-19.

[8] Zhou Zicheng.Research status of on water cooled chillers(1) [J].Refrigeration,2012,31(2):26-33.

[9] Zhou Zicheng.Research status of on water cooled chillers(2)[J].Refrigeration,2012,31(1):29-36.

[10] Eberhard Wobst, Nikoli kalitzin, Rainer Apley. Turbo water chiller with water as refrigerant [J]. International Compressor Engineering Conference at Purdue, 2004: 12-15.

[11] Ali Kilicarslan, Nobert Muller. A comparative study of water as a refrigerant with some current refrigerants [J]. International Journal of Energy Research, 2005, 29: 917-959.

[12] D.Van Orshoven,W.A Beckman.An investigation of water as the refrigerant[J].Journal of Energy Resources Technology, 1993: 257-263.

[13] Brandon F. Lachner,Gregory F. Nellis,Douglas T. Reindl.The commercial feasibility of water vapor as a refrigerant [J].International Journal of Refrigeration, 2007, 30: 699-708.

[14] Nathan Holden,Norbert Muller.Wheel diameter design for a water vapor (R-718) axial compressor [R].International Mechanical Engineering and Exposition. 2006.

[15] Qubo Li,Nobert Muller.Numerical simulation of novel axial impeller patterns to compress water vapor as refrigerant.[J] Energy, 2011. 36: 2773-2781. 